\title{
Propagation of guided cold atoms
}

\author{
T. Lahaye *, P. Cren, C. Roos, D. Guéry-Odelin \\ Laboratoire Kastler Brossel ${ }^{1}$, Ecole Normale Supérieure, 24 Rue Lhomond, F-75231 Paris Cedex 05, France
}

\begin{abstract}
In this article we focus on the propagation of a beam of particles guided by a transversely confining potential. We consider different regimes. In the classical regime, we describe the beam by means of a set of hydrodynamic-like equations. We apply this formalism in order to investigate two practical ways for increasing the collision rate: by using a constriction or by tilting the guide. A high enough collision rate is indeed the most crucial prerequisite for reaching the quantum degenerate regime by means of evaporative cooling. In the quantum regime, we study the propagation of bosonic atoms through a constriction in two opposite regimes: the collision-less one and the Thomas-Fermi one.
\end{abstract}

(C) 2003 Elsevier B.V. All rights reserved.

PACS: 03.75.Fi; 05.30.Jp; 67.40.Db

Keywords: Atom waveguide; Quantum-degenerate beams; Constriction

\section{Introduction}

So far, Bose-Einstein condensates have only been produced in a pulsed mode. To this respect, the achievement of a continuous beam operating in the quantum degenerate regime remains a real challenge. This would be the matter wave equivalent of a $\mathrm{cw}$ monochromatic laser and it would allow for unprecedented performance in terms of focalization or collimation. Recently the MIT group has demonstrated the periodic replenishment of an optical trap [1]. This technique can therefore be used in future experiments in combination with an appropriate outcoupler to continuously release atoms from the trap, and hence to obtain a truly cw atom laser.

Another possible way to achieve this goal has been studied theoretically in [2]. In this proposal, a non-degenerate, but already slow and cold beam of particles, is injected into a magnetic guide

* Corresponding author. Tel.: +33-1-44-32-3497.

E-mail address: thierry.lahaye@lkb.ens.fr (T. Lahaye).

${ }^{1}$ Unité de Recherche de l'Ecole normale supérieure et de l'Université Pierre et Marie Curie, associée au CNRS. 
[3-10] where transverse evaporation takes place. If the elastic collision rate is large enough, efficient evaporative cooling will lead to quantum degeneracy after a propagation length in the guide compatible with experimental constraints (i.e. a few meters). The continuous and pulsed loading of a slow and cold atomic beam into a magnetic guide has been recently demonstrated experimentally [11]. However, the collision rate is not sufficient so far to initiate the evaporative cooling stage.

In this article, we start with summarizing the experimental techniques already developed for atom guiding. To achieve conditions favorable to evaporative cooling one has to maximize the collision rate. For that purpose, we describe quantitatively two possible manipulations of a guided thermal beam: constriction and tilting of the guide. We then study the role of interactions on a beam of Bose-condensed atoms propagating through a constriction.

\section{Atom waveguide}

In recent years, many experimental groups have designed both macroscopic and microscopic guides for transporting laser-cooled atoms. In those guides, the confinement is provided by means of conservative forces. So far the dipole and magnetic forces have been used.

In past years, atomic transport by means of hollow optical fibers has been achieved [12]. In this scheme atoms are guided by the optical dipole force. However, the heating of atoms through intensity fluctuations and spontaneous emission may be a problem.

An alternative way consists in using magnetic guides. For alkali atoms, magnetic forces are strong since the magnetic moment $\mu$ is of the order of the Bohr magneton $\mu_{\mathrm{B}}$. As the thermal energy of laser-cooled alkali atoms (micro-Kelvin range) is generally much less than the groundstate hyperfine splitting ( $1 \mathrm{GHz}$ corresponds to $48 \mathrm{mK}$ ), we can assume that the field explored in the guide produces a linear Zeeman shift proportional to the hyperfine $g$-factor $g_{\mathrm{F}}$. We also assume that the the spin adiabatically follows the magnetic field, yielding an effective potential $U=g_{\mathrm{F}} m_{\mathrm{F}} \mu_{\mathrm{B}}|B|$. Depending on the atom's magnetic sublevel $m_{\mathrm{F}}$ (positive or negative), atoms are attracted towards or repelled from a magnetic extremum. Actually, Maxwell's equations for magnetostatics only allow for the realization of a minimum of static magnetic fields in vacuum [13]. Therefore, only low-field seekers can be trapped with inhomogeneous static magnetic fields. As a consequence, the atoms are not trapped in the energetically lowest state. This effect and the three-body recombination are responsible for the metastability of the confined gas.

Quadrupole guides can be produced by means of four wires or tubes equally spaced on a cylinder of radius $R$ and carrying currents $+I$ and $-I$ alternatively. The magnetic field is quadrupolar and is well approximated by the linear form: $B\left(r_{\perp}\right)=b^{\prime} r_{\perp}$ where $r_{\perp}=\left(x^{2}+y^{2}\right)^{1 / 2}$ is the distance from the $z$-axis and $b^{\prime} \propto I / R^{2}$ the strength of the magnetic gradient. In this configuration, atoms with low angular momentum are not stable against spin flips. To avoid this loss mechanism, one superimposes a bias field $B_{0}$ along the axis of the guide. Note that in this case the field strength no longer varies linearly with radius but is given by

$$
B\left(r_{\perp}\right)=\sqrt{B_{0}^{2}+b^{\prime 2} r_{\perp}^{2}} \simeq B_{0}+\frac{b^{\prime 2} r_{\perp}^{2}}{2 B_{0}} .
$$


The Taylor expansion around the center yields a quadratic variation of the field strength. The potential experienced by the atoms is therefore harmonic provided that their temperature $T$ fulfills the condition $k_{\mathrm{B}} T \ll \mu B_{0}$. A constriction in the potential can be created by reducing the spacing between the wires of the guide and/or decreasing the longitudinal bias field.

\section{Increasing the collision rate of a classical gas}

As mentioned in Section 1, the elastic collision rate $\Gamma_{\text {coll }}$ in the sample must be high enough at the entrance of magnetic guide to start an efficient evaporative cooling [2]. We study in this section two ways of increasing $\Gamma_{\text {coll }}$ in the classical regime for a beam of particles propagating through the guide. Both methods ensure the conservation of phase space density. The first one consists in a constriction of the transverse confining potential, while the second relies on gravity to slow down the beam by tilting the guide with respect to the horizontal plane.

\subsection{Constriction}

\subsubsection{Single particle}

Before studying the physics of a beam in the hydrodynamic regime, it is worthwhile to examine the one-particle motion in a constricted guide, in order to gain physical insight. We therefore write the equation of motion along $z$ for a particle with mass $m$ in the power law transverse potential $U(x, y, z)=m \lambda(z) r_{\perp}^{\gamma}$ where $\lambda(z)$ denotes the $z$-dependent strength of the transverse confinement,

$$
\frac{\mathrm{d}^{2} z}{\mathrm{~d} t^{2}}=-r_{\perp}^{\gamma} \frac{\mathrm{d} \lambda}{\mathrm{d} z} .
$$

Eq. (2) shows that the constriction induces a transfer of longitudinal energy into the transverse motion. For a smooth constriction, the motion is adiabatic [14] and consequently the quantity $E_{\perp} \lambda^{-2 /(2+\gamma)}$ is constant, where $E_{\perp}$ denotes the transverse energy. As the total energy $E=E_{\perp}+m \dot{z}^{2} / 2$ is also conserved, the decrease in longitudinal velocity can easily be related to the increase in $\lambda$. We notice that the effect of the constriction is more dramatic for particles having a high transverse energy, and is absent for a particle propagating along the axis.

\subsubsection{Hydrodynamic regime}

We now assume that there are enough collisions to ensure local thermodynamical equilibrium. From Appendix A we deduce the equations governing the beam temperature $T(z, t)$, linear density $n(z, t)$ and mean longitudinal velocity $u(z, t)$ :

$$
\begin{aligned}
& \frac{\partial n}{\partial t}+\frac{\partial}{\partial z}[n u]=0, \\
& \frac{\partial}{\partial t}[n u]+\frac{\partial}{\partial z}\left[\frac{n k_{\mathrm{B}} T}{m}+n u^{2}\right]+\frac{2 n k_{\mathrm{B}} T}{m \gamma \lambda} \frac{\mathrm{d} \lambda}{\mathrm{d} z}=0, \\
& \frac{\partial}{\partial t}\left[\left(\frac{3}{2}+\frac{2}{\gamma}\right) n k_{\mathrm{B}} T+\frac{m n u^{2}}{2}\right]+\frac{\partial}{\partial z}\left[\left(\frac{5}{2}+\frac{2}{\gamma}\right) n u k_{\mathrm{B}} T+\frac{m n u^{3}}{2}\right]=0 .
\end{aligned}
$$


We now study the stationary regime. The previous equations become

$$
\begin{aligned}
& n u=\phi, \\
& \frac{\mathrm{d}}{\mathrm{d} z}\left[\frac{k_{\mathrm{B}} T}{u}+m u\right]+\frac{2 k_{\mathrm{B}} T}{\gamma u \lambda} \frac{\mathrm{d} \lambda}{\mathrm{d} z}=0, \\
& \left(\frac{5}{2}+\frac{2}{\gamma}\right) k_{\mathrm{B}} T+\frac{m u^{2}}{2}=\mu,
\end{aligned}
$$

where $\phi$ and $\mu$ are constants. Eq. (3) corresponds to the conservation of the number of particles, Eq. (4) of momentum along $z$ and Eq. (5) of enthalpy. After some algebra one obtains the relation between the strength of the potential $\hat{\lambda} \equiv \lambda(z) / \lambda(0)$ normalized to its initial value and the reduced temperature $\widehat{T} \equiv T(z) / T(0)$ :

$$
\hat{\lambda}=\widehat{T}^{\gamma(\delta-2) / 4}\left(1-\frac{\delta}{\eta_{0}}(\widehat{T}-1)\right)^{\gamma / 4},
$$

where we have introduced the parameters $\delta \equiv 5+4 / \gamma$ and $\eta_{0}=m u(0)^{2} /\left(k_{\mathrm{B}} T(0)\right)$ which compares the initial kinetic and thermal energies. For typical experimental values [11], one has $\eta_{0} \gg 1$ and (6) yields

$$
\widehat{T} \simeq \hat{\lambda}^{4 /(\gamma(\delta-2))} .
$$

As the normalized collision rate $\widehat{\Gamma} \equiv \Gamma_{\text {coll }}(z) / \Gamma_{\text {coll }}(0)$ increases as $\widehat{\Gamma} \sim \widehat{T}^{2}$, one deduces the scaling law $\widehat{\Gamma} \sim \hat{\lambda}^{8 /(\gamma(\delta-2))}$, which shows that moderate constrictions (for example by a factor of 4 ) can increase the collision rate by a large amount (nearly one order of magnitude for an harmonic transverse confinement).

\subsection{Tilted guide}

We now consider that the guide axis $z$ is tilted by an angle $\alpha$ with respect to the horizontal plane. The strength $\lambda$ of the transverse confining potential is assumed constant. By tilting the guide, the mean velocity of the beam decreases while the linear density and the temperature increase, resulting in an increase of the collision rate. Following the same procedure as the one developed in Appendix A, we get, in the stationary regime, the following equations:

$$
\begin{aligned}
& n u=\phi, \\
& \frac{\mathrm{d}}{\mathrm{d} z}\left[m u+\frac{k_{\mathrm{B}} T}{u}\right]+\frac{m g \sin \alpha}{u}=0, \\
& \left(\frac{2}{\gamma}+\frac{5}{2}\right) k_{\mathrm{B}} T+\frac{m u^{2}}{2}+m g z \sin \alpha=\mu,
\end{aligned}
$$

where $g$ is the acceleration of gravity. This set of equations can be solved analytically, one deduces the relation between the reduced temperature $\widehat{T}$ and the reduced velocity of the beam $\hat{u}=u(z) / u(0): \widehat{T}=u^{2 /(2-\delta)}$. We also establish the relation between the collision rate and the coordinate $\hat{z} \equiv z / z_{0}$ with $z_{0}=u_{0}^{2} /(g \sin \alpha)$, and the link between the parameter $\hat{\eta}=m u^{2} /\left(\eta_{0} k_{\mathrm{B}} T\right)=$ $\hat{u}^{2} / \widehat{T}$ and $\hat{z}$ : 

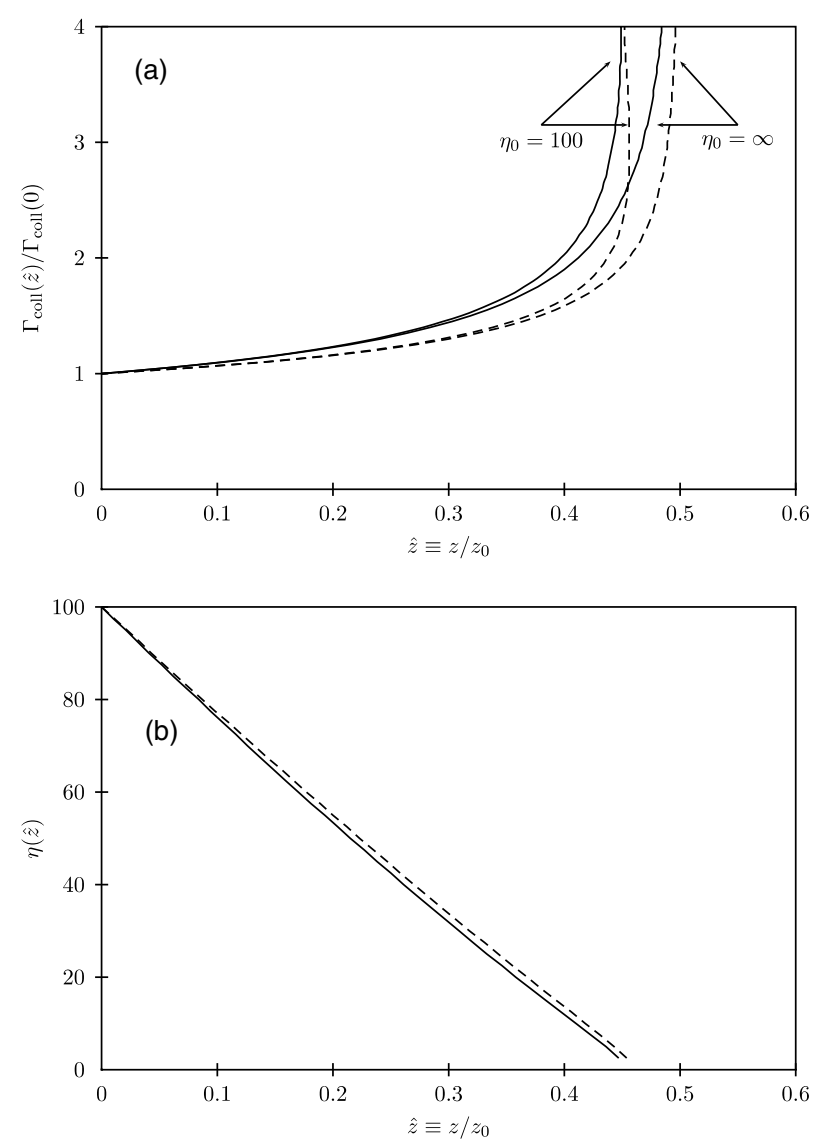

Fig. 1. (a) Collision rate normalized at its initial value $\widehat{\Gamma}(\hat{z})=\Gamma_{\text {coll }}(\hat{z}) / \Gamma_{\text {coll }}(0)$ as a function of the reduced longitudinal coordinate $\hat{z}$ for a tilted guide, for $\eta_{0}=100$ and $\eta_{0}=\infty$. The dashed curves correspond to a linear transverse confinement $(\gamma=1)$ and the solid lines to a harmonic transverse confinement $(\gamma=2)$. (b) Parameter $\eta \equiv m u^{2}(\hat{z}) /\left(k_{\mathrm{B}} T(\hat{z})\right)$ as a function of $\hat{z}$, for an initial value of 100 , for linear (--) and harmonic (-) transverse confinement.

$$
\begin{aligned}
& \hat{z}(\widehat{\Gamma})=\frac{\delta+\eta_{0}}{2 \eta_{0}}-\frac{\delta}{2 \eta_{0}} \widehat{\Gamma}^{1 / 2}-\frac{1}{2} \widehat{\Gamma}^{(2-\delta) / 2}, \\
& \hat{z}(\hat{\eta})=\frac{\delta+\eta_{0}}{2 \eta_{0}}-\frac{\delta}{2 \eta_{0}} \hat{\eta}^{1 /(1-\delta)}-\frac{1}{2} \hat{\eta}^{(2-\delta) /(1-\delta)} .
\end{aligned}
$$

We recall that for a particle with initial velocity $u_{0}$ moving in this potential, the turning point corresponds to $\hat{z}=1 / 2$. Fig. 1(a) shows the gain in the collision rate as a function of $\hat{z}$ for 2 values of the parameter $\eta_{0}=100$ and $\eta_{0}=\infty$ and for an harmonic $(\gamma=2)$ and a linear $(\gamma=1)$ confinement. Along the propagation through the tilted guide, the temperature $T$ increases and the mean velocity $u$ decreases resulting in a strong reduction of the parameter $\eta(z)$ as a function of $z$ (see Fig. 1(b)).

In this section, we have described the propagation of a beam of classical particles through a constriction and a tilted guide by means of a set of hydrodynamic-like equations. We have used this formalism to investigate two practical ways for increasing the collision rate. Indeed those 
results are valid for a beam of bosonic particles. We recall that a high collision rate is the most crucial requirement for reaching the quantum degenerate regime by means of evaporative cooling [2]. The remainder of the paper is devoted to the quantum degenerate regime. We focus on the propagation through a constriction, and analyze the role of interactions.

\section{Constriction of a quantum beam}

For atomic waveguide geometries with very small transverse dimensions as now experimentally demonstrated [3-10], the quantum nature of atoms starts to influence the dynamics. This problem has first been investigated in the context of quantized-conductance in a two-dimensional electron gas [15]. In those models, the conducting properties of a nanoscopic constriction are investigated in the free-electron approximation with hard-walls boundary conditions. In this section, we address the corresponding problem for a beam of Bose-condensed atoms confined in the transverse direction. In the following, we restrict our study only to harmonic confinement for the sake of simplicity. As for the classical case of the previous section, we model the constriction by a dependence of the transverse frequency with the longitudinal coordinate. The potential experienced by the atoms is therefore of the form $U(x, y, z)=m \omega(z)^{2}\left[x^{2}+y^{2}\right] / 2$. We begin with the case of an ideal beam, and then consider the role played by interactions for the propagation through a constriction.

\subsection{The ideal Bose-condensed beam}

In this paragraph we study the propagation of an ideal Bose-condensed beam. For this purpose, we solve the stationary Schrödinger equation by means of the adiabatic basis (the harmonic oscillator's one with an angular frequency that depends on the longitudinal coordinate $z$ ). Details of this lengthy but straightforward calculation can be found in the Appendix B. If $k$ denotes the incoming longitudinal wave vector, the local wave vector $k_{0,0}$ for atoms in the transverse ground state within the adiabatic approximation reads (see Eq. (B.2)):

$$
k_{0,0}^{2}=k^{2}-\frac{1}{\sigma^{2}},
$$

$\sigma$ being the local harmonic oscillator length $\sigma(z)=(\hbar / m \omega(z))^{1 / 2}$. The physical picture is quite clear: the constriction induces an augmentation in the zero-point energy for the transverse degrees of freedom. As the total energy of the atom is conserved, the longitudinal kinetic energy decreases. Actually, one recovers, in the quantum domain, the transfer of kinetic energy from longitudinal to transverse degrees of freedom as already pointed out for the classical dynamics. However, the constriction modifies the propagation even for particles in the transverse ground state because of the finite size of the transverse wave function.

\subsection{The role of interactions}

We investigate in this paragraph the role of interactions on the propagation through a constriction of a beam of interacting Bose-condensed atoms. The main difficulty arises from the non- 
linearity of the Schrödinger equation due to the mean field interaction term. The strength of the interaction is proportional to the scattering length $a$ [16]. By assuming that the transverse dimension of the cloud has the local equilibrium shape adapted to the local number of particle per unit length, the problem becomes one-dimensional and one can introduce a solution for the wave function of the form $\psi(\mathbf{r}, t)=f(z, t) g(x, y ; n)$ in the variational procedure [17], where $n$ is the local density of particles per unit length. By writing the longitudinal part of the wave function $f$ in the phase and modulus form $f \equiv \sqrt{n} \mathrm{e}^{\mathrm{i} \varphi}$, we introduce the longitudinal velocity field defined by $u \equiv(\hbar / m) \partial \varphi / \partial z$.

After some algebra (see Appendix C), one gets the stationary hydrodynamic equations for the propagation of a beam of interacting Bose-condensed particles:

$$
\begin{aligned}
& n u=\phi, \\
& \tilde{\mu}+\frac{1}{2} m u^{2}-\frac{\hbar^{2}}{2 m \sqrt{n}} \frac{\partial^{2} \sqrt{n}}{\partial z^{2}}=c,
\end{aligned}
$$

where $\tilde{\mu}$ is an effective chemical potential, and $\phi$ and $c$ two constants depending on the initial conditions. Eq. (12) reflects the conservation of the flux while Eq. (13) accounts for the conservation of energy.

As a first example, from Eqs. (12) and (13) we recover the results of the previous section for a perfect gas for which $a=0$ and $\tilde{\mu}=\hbar \omega$ in a smooth constriction i.e. such that the last term of the 1.h.s of (13) is negligible. In the following, we apply this set of equations in the weak-coupling limit $(n a \ll 1)$ and in the Thomas-Fermi limit $(n a \gg 1)$. For those two limits, the value of $\tilde{\mu}$ differs (see Appendix C). It is convenient to introduce the dimensionless notations: $\hat{u} \equiv u(z) / u(0), \hat{z} \equiv z / \sigma(0)$, $\hat{\omega} \equiv \omega(z) / \omega(0), \alpha \equiv m u^{2}(0) /(\hbar \omega(0))$ and $\beta \equiv \sqrt{n a}=\sqrt{a \phi / u(0)}$. The combination of Eqs. (12) and (13) leads to the relation between the reduced velocity $\hat{u}$ and the rate of compression $\hat{\omega}$ as a function of the dimensionless parameters.

In the weak-coupling limit that corresponds to the low density regime $(\beta \ll 1)$ we find:

$$
\frac{\mathrm{d}^{2} \hat{u}}{\mathrm{~d} \hat{z}^{2}}=\frac{3}{2 \hat{u}}\left(\frac{\mathrm{d} \hat{u}}{\mathrm{~d} \hat{z}}\right)^{2}-2 \alpha \hat{u}\left(\hat{u}^{2}-1\right)+4 \hat{u}(1-\hat{\omega})+8 \hat{u} \beta^{2}(1-\hat{\omega} / \hat{u})
$$

while in the opposite regime, commonly called the Thomas-Fermi regime, we have

$$
\frac{\mathrm{d}^{2} \hat{u}}{\mathrm{~d} \hat{z}^{2}}=\frac{3}{2 \hat{u}}\left(\frac{\mathrm{d} \hat{u}}{\mathrm{~d} \hat{z}}\right)^{2}-2 \alpha \hat{u}\left(\hat{u}^{2}-1\right)+8 \beta(\hat{u}-\hat{\omega} \sqrt{\hat{u}}) .
$$

In the following, we consider a linear increase of the transverse confining frequency: $\hat{\omega}=1+\gamma \hat{z}$. The results of the numerical integration of Eqs. (14) and (15) are plotted on Fig. 2, for the following values of the dimensionless parameters: $\alpha=10^{4}, \gamma=10^{-4}, \beta$ having the value 10 in the Thomas-Fermi regime, and 0.1 in the weak-coupling regime. The large constriction ratios implied by $\gamma=10^{-4}$ can be achieved on microchips [18]. For a given compression rate, the reduction of the velocity for the propagation through a constriction is dramatically magnified in the interacting case. It reflects the fact that the ground state wave function has a much broader transverse profile because of repulsive interactions $(\sqrt{n a} \gg 1)$. As already emphasized in Section 3.1.1, the larger the transverse size of the beam, the higher the coupling between longitudinal and transverse degrees of 


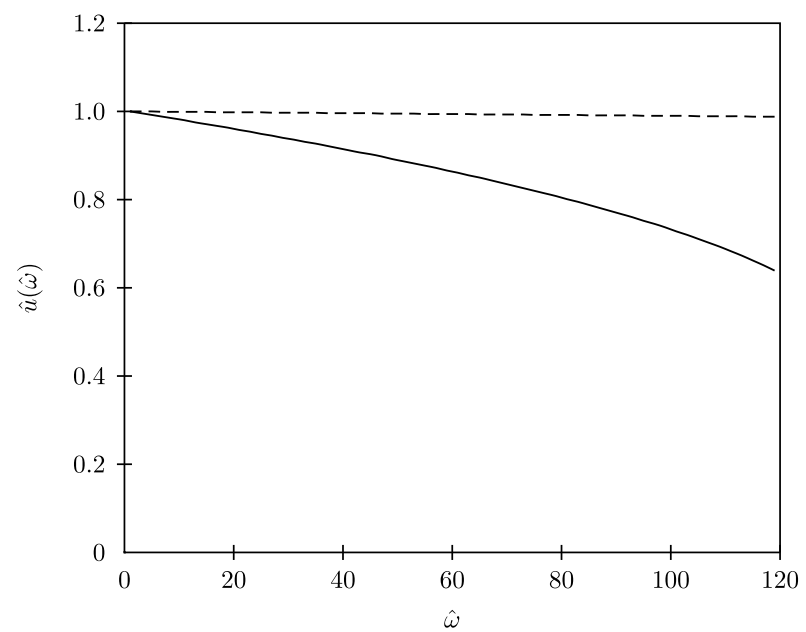

Fig. 2. Longitudinal velocity $\hat{u} \equiv u / u_{0}$ as a function of the constriction ratio $\hat{\omega}$ for a quantum beam in the weakcoupling regime $(--, \beta=0.1)$ and in the Thomas-Fermi regime $(-, \beta=10)$ for $\alpha=10^{4}$ and $\gamma=10^{-4}$. In the ThomasFermi regime, the repulsive interactions lead to a strong coupling between longitudinal and transverse degrees of freedom resulting in a larger reduction of the beam velocity through the constriction.

freedom. We point out that this magnification of the coupling between degrees of freedom induced by interactions may have to be taken into account for interferometers based on guided matter waves [19].

\section{Conclusion}

In this article, we have investigated quantitatively two possible ways (by compression and tilting of the guide) of increasing the collision rate of a thermal guided beam while keeping the phase space density constant. Those results are important from an experimental point of view since as soon as the collision rate is high enough, one can implement evaporative cooling as suggested in [2] to reach the quantum degeneracy regime. Those results have been obtained under the hydrodynamic formalism. In future work, we intend to the study the case where the collision rate is not sufficient to ensure the local equilibrium requirement for hydrodynamics. We have also carried out the calculation for the propagation of a beam of bosons through a constriction in the degenerate regime in presence or not of interactions between particles. Strictly speaking, those calculations are valid at $T=0$. Actually, the propagation of a beam of bosons through a constriction below the critical temperature can be regarded as a two-fluid problem. To address this problem completely, one would have to take into account the interaction between the thermal and the condensed part.

\section{Acknowledgements}

We thank Jean Dalibard and Johnny Vogels for a careful reading of the manuscript. C.F. Roos acknowledges support from the European Union (contract HPMFCT-2000-00478). This work 
was supported by the Bureau National de la Métrologie, the Délégation Générale de l'Armement, the Centre National de la Recherche Scientifique and the Région Ile de France.

\section{Appendix A. Theoretical framework for guided beams in classical regime}

We consider a gas of particles with mass $m$ confined by a static, velocity-independent potential $U(\mathbf{r})$. In this appendix we derive the classical equations for a beam of particles confined in a cylindrical geometry. For that purpose, we start with the classical Boltzmann equation:

$$
\frac{\partial f}{\partial t}+\mathbf{v} \cdot \frac{\partial f}{\partial \mathbf{r}}-\frac{1}{m} \frac{\partial U}{\partial \mathbf{r}} \cdot \frac{\partial f}{\partial \mathbf{v}}=I_{\text {coll }}[f]
$$

where $f(\mathbf{r}, \mathbf{v}, t)$ is the distribution function and $I_{\text {coll }}[f]$ is collision term. The density $\rho$ is obtained from the distribution by integration over velocity:

$$
\rho(\mathbf{r}, t)=\int d^{3} v f(\mathbf{r}, \mathbf{v}, t) .
$$

\section{A.1. Reduction of dimensionality}

We present the formulation of conservation equations for cylindrical geometries. We choose the longitudinal axis along $z$. In order to get hydrodynamic-like equations, we assume that the distribution function $f$ is given by a local Maxwell-Boltzmann distribution of the form:

$$
f(\mathbf{r}, \mathbf{v}, t) \propto \exp \left\{-\frac{m\left(\mathbf{v}-u(z, t) \mathbf{e}_{z}\right)^{2}}{2 k_{\mathrm{B}} T(z, t)}-\frac{U(x, y, z)}{k_{\mathrm{B}} T(z, t)}\right\} .
$$

In the ansatz (A.3) we assume that the velocity field is purely longitudinal, and that all macroscopic quantities depend only on $z{ }^{2}$ We introduce the linear density $n$ defined by:

$$
n(z, t)=\int \mathrm{d} x \mathrm{~d} y \mathrm{~d}^{3} v f(\mathbf{r}, \mathbf{v}, t) .
$$

The conservation of the number of particles is obtained by taking the average of (A.1) over the transverse coordinates and the velocity components: $\int[A .1] \mathrm{d} x \mathrm{~d} y \mathrm{~d}^{3} v$ :

$$
\frac{\partial n}{\partial t}+\frac{\partial}{\partial z}\left(n\left\langle v_{z}\right\rangle\right)=0
$$

where $\langle\cdots\rangle$ denotes the following normalized average:

$$
\langle A\rangle \equiv \frac{1}{n(z)} \int \mathrm{d} x \mathrm{~d} y \mathrm{~d}^{3} v A f(\mathbf{r}, \mathbf{v}, t)
$$

\footnotetext{
${ }^{2}$ The set of hydrodynamic-like equations derived in Appendix A can be recovered directly from hydrodynamic equations with the proper choice for the ansatz. Actually, the radial dependence of the velocity field has to be taken into account even if it does not appear explicitly in the 1D equations.
} 
The axial momentum current equation is deduced from the average of the on-axis component of the velocity $\int[A .1] v_{z} \mathrm{~d} x \mathrm{~d} y \mathrm{~d}^{3} v$ :

$$
\frac{\partial}{\partial t}\left(n\left\langle v_{z}\right\rangle\right)+\frac{\partial}{\partial z}\left(n\left\langle v_{z}^{2}\right\rangle\right)+\frac{n}{m}\left\langle\frac{\partial U}{\partial z}\right\rangle=0 .
$$

The conservation of energy is obtained from the average of the one-particle hamiltonian $H(\mathbf{r}, \mathbf{v})=m v^{2} / 2+U(\mathbf{r}), \int[A .1] H(\mathbf{r}, \mathbf{v}) \mathrm{d} x \mathrm{~d} y \mathrm{~d}^{3} v$

$$
\frac{\partial}{\partial t}(n\langle H\rangle)+\frac{\partial}{\partial z}\left(n\left\langle v_{z}\right\rangle\langle H\rangle\right)+\frac{m}{2} \frac{\partial}{\partial z}\left(n\left(\left\langle v_{z}^{3}\right\rangle-\left\langle v_{z}\right\rangle\left\langle v_{z}^{2}\right\rangle\right)\right)=0
$$

\section{A.2. Application to a power law potential for a stationary flow}

We consider in this section that the transverse confining potential is given by a power law of exponent $\gamma$ with a $z$-dependent strength $\lambda(z)$ :

$$
U(x, y, z)=m \lambda(z) r_{\perp}^{\gamma}
$$

where $r_{\perp}=\left(x^{2}+y^{2}\right)^{1 / 2}$ denotes the radial distance from the guide axis.

Furthermore, we define the mean velocity of the beam by $u \equiv\left\langle v_{z}\right\rangle$, and calculate with the ansatz (A.3) the following useful quantities: $\left\langle v_{z}^{2}\right\rangle=u^{2}+k_{\mathrm{B}} T / m$ and $\left\langle v_{z}^{3}\right\rangle=u^{3}+3 u k_{\mathrm{B}} T / m$. One also readily calculates the average values $\langle U\rangle=2 k_{\mathrm{B}} T / \gamma,\langle H\rangle=(2 / \gamma+3 / 2) k_{\mathrm{B}} T+m u^{2} / 2$. The onedimensional hydrodynamic equations (A.5)-(A.7) then simplify considerably. In the stationary regime that we shall consider hereafter, they can be recast in the form:

$$
\begin{aligned}
& n u=\phi, \\
& \frac{\mathrm{d}}{\mathrm{d} z}\left[\frac{k_{\mathrm{B}}}{u} T+m u\right]+\frac{2 k_{\mathrm{B}} T}{\gamma \lambda u} \frac{\mathrm{d} \lambda}{\mathrm{d} z}=0, \\
& \left(\frac{2}{\gamma}+\frac{5}{2}\right) k_{\mathrm{B}} T+\frac{m u^{2}}{2}=\mu .
\end{aligned}
$$

An interesting feature of this set of equations is the conservation of phase-space density $D \equiv \rho \lambda_{\mathrm{dB}}^{3}$, where $\lambda_{\mathrm{dB}}=h / \sqrt{2 \pi m k_{\mathrm{B}} T}$ is the thermal de Broglie wavelength and $\rho$ the 3D particle density. Indeed, for the power-law potential (A.8), $D$ scales with the four parameters $u, T, \lambda$ and $\gamma$ as: $D \sim \lambda^{2 / \gamma} u^{-1} T^{-2 / \gamma-3 / 2}$. One can easily check that the set of equations (A.9) implies that

$$
\frac{\mathrm{d} D}{\mathrm{~d} z}=0
$$

The collision rate $\Gamma_{\text {coll }}$ scales as $\rho T^{1 / 2}$, thus the ratio $\widehat{\Gamma}=\Gamma_{\text {coll }}(z) / \Gamma_{\text {coll }}(0)$ scales as $(T(z) / T(0))^{2}$.

\section{Appendix B. Compression of an ideal quantum gas}

In this appendix, we address the problem of the propagation of a quantum gas in a harmonic waveguide with a constriction. The potential experienced by the atoms reads: $U(x, y, z)=$ 
$m \omega^{2}(z)\left(x^{2}+y^{2}\right) / 2, \omega(z)$ being the $z$-dependent angular frequency. We introduce the harmonic oscillator length $\sigma(z)=(\hbar / m \omega(z))^{1 / 2}$, and denote its first and second derivatives with respect to $z$ by $\sigma^{\prime}$ and $\sigma^{\prime \prime}$. For a theoretical description, we resort to the study of the stationary solutions of the Schrödinger equation [15]. A general wave function can be expanded on the adiabatic basis as $|\Psi\rangle=\sum_{n_{x}, n_{y}} \alpha_{n_{x}, n_{y}}(z)\left|n_{x}(z)\right\rangle \otimes\left|n_{y}(z)\right\rangle$, where $\left\{\left|n_{i}(z)\right\rangle\right\}$ denotes the local 1D harmonic basis for the $i$-direction with a $z$-dependence in angular frequency. Inserting $|\Psi\rangle$ into the Schrödinger equation with stationary energy $E \equiv \hbar^{2} k^{2} / 2 m$ and projecting on the mode $\left|m_{x}(z)\right\rangle \otimes\left|m_{y}(z)\right\rangle$ yields the following set of coupled linear equations:

$$
\begin{aligned}
\frac{\mathrm{d}^{2} \alpha_{m_{x}, m_{y}}}{\mathrm{~d} z^{2}}+k_{m_{x}, m_{y}}^{2} \alpha_{m_{x}, m_{y}}= & -\sum_{n_{x} \neq m_{x}}\left(2 \frac{\mathrm{d} \alpha_{n_{x}, m_{y}}}{\mathrm{~d} z} A_{m_{x}, n_{x}}+\alpha_{n_{x}, m_{y}} B_{m_{x}, n_{x}}\right) \\
& -\sum_{n_{y} \neq m_{y}}\left(2 \frac{\mathrm{d} \alpha_{m_{x}, n_{y}}}{\mathrm{~d} z} A_{m_{y}, n_{y}}+\alpha_{m_{x}, n_{y}} B_{m_{y}, n_{y}}\right)+2 \sum_{\substack{n_{x} \neq m_{x} \\
n_{y} \neq m_{y}}} \alpha_{n_{x}, n_{y}} A_{m_{x}, n_{x}} A_{m_{y}, n_{y}},
\end{aligned}
$$

where we have defined the matrix elements $A_{m_{i}, n_{i}}=\left\langle m_{i}|\partial / \partial z| n_{i}\right\rangle, B_{m_{i}, n_{i}}=\left\langle m_{i}\left|\partial^{2} / \partial z^{2}\right| n_{i}\right\rangle$, and the local wave number $k_{m_{x}, m_{y}}$

$$
k_{m_{x}, m_{y}}^{2}=k^{2}-\frac{\sigma^{\prime 2}}{2 \sigma^{2}}\left(2+m_{x}+m_{y}+m_{x}^{2}+m_{y}^{2}\right)-\frac{1+2 m_{x}+2 m_{y}}{\sigma^{2}} .
$$

The matrix elements can be readily calculated:

$$
\begin{aligned}
& A_{m_{i}, n_{i}}=\sigma^{\prime}\left\langle m_{i}\left|a_{i}^{\dagger 2}-a_{i}^{2}\right| n_{i}\right\rangle / 2 \sigma, \\
& B_{m_{i}, n_{i}}=\frac{1}{4 \sigma^{2}}\left(\sigma^{\prime 2}\left\langle m_{i}\left|\left[a_{i}^{\dagger 2}-a_{i}^{2}\right]^{2}\right| n_{i}\right\rangle-2\left(\sigma \sigma^{\prime \prime}-\sigma^{\prime 2}\right)\left\langle m_{i}\left|a_{i}^{\dagger 2}-a_{i}^{2}\right| n_{i}\right\rangle\right),
\end{aligned}
$$

where $a_{i}^{\dagger}$ and $a_{i}$ are the creation and annihilation operators for the considered harmonic basis.

Eq. (B.1) shows that each mode experiences a different potential barrier. In particular, $k_{m_{x}, m_{y}}(z)$ decreases in for a propagation through a constriction since the ground state energy increases in this case. From the above analysis we can conclude that an incident wave initially in the transverse ground state remains in this state if the adiabatic approximation criterium is fulfilled, namely $\left|\sigma^{\prime}\right| \ll 1$. The adiabatic approximation fails around the turning point if the constriction is such that the incident wave can be reflected. We note that if the condition of adiabatic approximation is not fulfilled, Eq. (B.1) allows one to evaluate the importance of inter-level scattering.

\section{Appendix C. Effective one-dimensional hydrodynamic equation in the quantum regime}

In this appendix, we derive the effective 1D hydrodynamic equations for a beam of Bosecondensed interacting atoms propagating through a guide. For this purpose, we recall the main steps of their derivations as presented in [17] by means of the standard variational procedure. This approach is valid under the dilute-gas assumption [16], namely $\bar{\rho} a^{3} \ll 1$ where $a$ is the scattering length and $\bar{\rho}$ the mean density. 


\section{C.1. Hydrodynamic equations}

The action $\mathscr{S}$ for the condensate wave function $\psi$ reads:

$$
\mathscr{S}=\int \mathrm{d} t \mathscr{E}\left[\psi, \psi^{*}\right]-\frac{\mathrm{i} \hbar}{2} \int \mathrm{d} t \mathrm{~d}^{3} r\left(\psi^{*} \frac{\partial \psi}{\partial t}-\psi \frac{\partial \psi^{*}}{\partial t}\right)
$$

where the energy functional $\mathscr{E}$ is given by

$$
\mathscr{E}\left[\psi, \psi^{*}\right]=\int \mathrm{d}^{3} r\left(\frac{\hbar^{2}}{2 m}|\nabla \psi|^{2}+\frac{2 \pi \hbar^{2} a}{m}|\psi|^{4}+U(\mathbf{r})|\psi|^{2}\right) .
$$

The fact that the transverse beam size is small compared with the other relevant length scales allows us to consider the density profile across the guide to have its equilibrium form appropriate to the local number of particles per unit length. As a consequence, the problem becomes essentially one dimensional. In this case, the wave function can be assumed to have the form: $\psi(\mathbf{r}, t)=f(z, t) g(x, y ; n)$ where $n$ is the local density of particles per unit length and $g$ the equilibrium wave function for the transverse motion. We emphasize that this ansatz does not correspond to a complete separability between the axial and transverse coordinates since $g$ depends on $z$ via the density per unit length $n$. In the following, we choose $g$ to be normalized to $\int|g|^{2} \mathrm{~d} x \mathrm{~d} y=1$, and therefore $|f|^{2}=n$.

The equations for $g$ and $f$ are obtained by minimization:

$$
\frac{\delta}{\delta g^{*}}\left(\mathscr{E}-\mu \int \mathrm{d}^{3} r|\psi|^{2}\right)=0 \quad \text { and } \quad \frac{\delta \mathscr{S}}{\delta f^{*}}=0,
$$

where $\mu$ is the chemical potential. After some algebra, one obtains the following set of equations:

$$
\begin{aligned}
& \left(-\frac{\hbar^{2}}{2 m} \Delta+U+\frac{4 \pi \hbar^{2} a}{m}|f g|^{2}\right) g=\tilde{\mu} g \\
& \mathrm{i} \hbar \frac{\partial f}{\partial t}=-\frac{\hbar^{2}}{2 m}\left(\frac{\partial^{2} f}{\partial z^{2}}-f \int|\nabla g|^{2} \mathrm{~d} x \mathrm{~d} y\right)+\frac{4 \pi \hbar^{2} a}{m} f|f|^{2} \int \mathrm{d} x \mathrm{~d} y|g|^{4}+f \int \mathrm{d} x \mathrm{~d} y U|g|^{2}
\end{aligned}
$$

where we introduce the effective chemical potential $\tilde{\mu}=\mu-\hbar^{2}|\partial f / \partial z|^{2} /\left(2 m|f|^{2}\right)$. To obtain the hydrodynamic-like equations, it is convenient to write $f$ in terms of a modulus and a phase: $f \equiv \sqrt{n} \mathrm{e}^{\mathrm{i} \varphi}$. The velocity field $u$ associated with the phase $\varphi$ reads $u \equiv(\hbar / m) \partial \varphi / \partial z$. From (C.4) and (C.5), one derives the equations for the density $n$ and the velocity $u$ :

$$
\begin{aligned}
& \frac{\partial n}{\partial t}+\frac{\partial}{\partial z}(n u)=0, \\
& m \frac{\partial u}{\partial t}=-\frac{\partial}{\partial z}\left(\tilde{\mu}+\frac{1}{2} m u^{2}-\frac{\hbar^{2}}{2 m} \frac{1}{\sqrt{n}} \frac{\partial^{2}(\sqrt{n})}{\partial z^{2}}\right) .
\end{aligned}
$$

The first equation is the usual equation of continuity, while the second is an Euler-like equation. In the adiabatic limit, the last term of (C.7) is negligible. 


\section{C.2. Determination of the chemical potential}

In the following, we derive the expression for the chemical potential in two opposite limits, namely the Thomas Fermi regime $(n a \gg 1)$ and the weak coupling limit $(n a \ll 1)$. We restrict our analysis to an harmonic trapping potential: $U(x, y, z)=m \omega^{2}\left(x^{2}+y^{2}\right) / 2$.

\section{C.2.1. The Thomas-Fermi regime}

In this large density limit ( $n a \gg 1$ ), the kinetic term in (C.4) can be neglected. The normalization condition on $g$ provides the relation between the effective chemical potential $\tilde{\mu}$ and the local density of particles per unit length: $\tilde{\mu}=2 \hbar \omega \sqrt{n a}$. If $\omega$ does not depend on $z$ or changes smoothly, $|\partial f / \partial z| \sim 0$ and $\tilde{\mu}=\mu$, we therefore recover the results of Refs. [17,20].

\section{C.2.2. The weak-interaction limit}

In the absence of interaction, $g_{0}$ has a Gaussian form, $\left|g_{0}\right|^{2}=\left(\pi \sigma^{2}\right)^{-1} e^{-\left(x^{2}+y^{2}\right) / \sigma^{2}}$ with $\sigma=\sqrt{\hbar / m \omega}$ and $\mu_{0}=\hbar \omega$. The low density limit corresponds to $n a \ll 1$, we consequently derive perturbatively from Eq. (C.4) the correction to the chemical potential:

$$
\mu=\mu_{0}+\delta \mu=\mu_{0}+\frac{4 \pi \hbar^{2} a}{m} n \int \mathrm{d} x \mathrm{~d} y\left|g_{0}\right|^{2}=\hbar \omega(1+2 a n) .
$$

\section{References}

[1] Chikkatur AP, Shin Y, Leanhardt AE, Kielpinski D, Tsikata E, Gustavson TL, et al. Science 2002;296:2193.

[2] Mandonnet E, Minguzzi A, Dum R, Carusotto I, Castin Y, Dalibard J. Eur Phys J D 2000;10:9.

[3] Schmiedmayer J. Phys Rev A 1995;52:R13-6.

[4] Denschlag J, Cassettari D, Schmiedmayer J. Phys Rev Lett 1999;82:2014.

[5] Goepfert A, Lison F, Schütze R, Wynands R, Haubrich D, Meschede D. Appl Phys B 1999;69:217.

[6] Key M, Hughes IG, Rooijakkers W, Sauer BE, Hinds EA, Richardson DJ, et al. Phys Rev Lett 2000;84:1371.

[7] Dekker NH, Lee CS, Lorent V, Thywissen JH, Smith SP, Drndic M, et al. Phys Rev Lett 2000;84:1124.

[8] Teo BK, Raithel G. Phys Rev A 2001;63:031402.

[9] Sauer JA, Barrett MD, Chapman MS. Phys Rev Lett 2001;87:270401.

[10] Hinds EA, Hughes IG. J Phys D: Appl Phys 1999;87:R119.

[11] Cren P, Roos CF, Aclan A, Dalibard J, Guéry-Odelin D. Eur Phys J D 2002;20:107.

[12] Renn MJ, Montgomery D, Vdovin O, Anderson DZ, Wieman CE, Cornell EA. Phys Rev Lett 1995;75:3253; Renn MJ, Donley EA, Cornell EA, Wieman CE, Anderson DZ. Phys Rev A 1996;53:R648; Renn MJ, Zozulya AA, Donley EA, Cornell EA, Anderson DZ. Phys Rev A 1997;55:3684; Ito H, Sakaki K, Ohtsu M, Jhe W. Appl Phys Lett 1997;70:2496.

[13] Wing WH. Prog Quant Electron 1984;8:181.

[14] Goldstein H. Classical Mechanics. 2nd ed. Reading, MA: Addison-Wesley; 1980.

[15] Glazman LI, Lesovik GB, Khmel'nitskii DE, Skekhter RI. J Exp Theor Phys Lett 1988;48:238; Yacoby A, Imry Y. Phys Rev B 1990;41:5341;

Stafford CA, Baeriswyl D, Bürki J. Phys Rev Lett 1997;79:2863;

Kassubek F, Stafford CA, Grabert H. Phys Rev B 1999;59:7560.

[16] Dalfovo F, Giorgini S, Pitaevskii L, Stringari S. Rev Mod Phys 1999;71:463. 
[17] Jackson AD, Kavoulakis GM, Pethick CJ. Phys Rev A 1998;58:2417.

[18] Ott H, Fortagh J, Schlotterbeck G, Grossmann A, Zimmermann C. Phys Rev Lett 2001;87:230401;

Hänsel W, Hommelhoff P, Hänsch TW, Reichel J. Nature 2001;413:498;

Haase A, Cassettari D, Hessmo B, Scmiedmayer J. Phys Rev A 2001;64:043405;

Vengalattore M, Rooijakkers W, Prentiss M. Phys Rev A 2002;66:053403.

[19] Berman P, editor. Atom Interferometry. New York: Academic Press; 1997;

Andersson E, Calarco T, Folman R, Andersson M, Hessmo B, Schmiedmayer J. Phys Rev Lett 2002;88:100401.

[20] Leboeuf P, Pavloff N. Phys Rev A 2001;64:033602. 